\title{
Boundary Conditions and Mode Jumping in the Buckling of a Rectangular Plate
}

\author{
David Schaeffer ${ }^{1 \star}$ and Martin Golubitsky ${ }^{2 \star \star}$ \\ Department of Mathematics, ${ }^{1}$ Duke University, Durham, NC 27706, and ${ }^{2}$ Arizona State University, \\ Tempe, AZ 85281 USA
}

\begin{abstract}
We show that mode jumping in the buckling of a rectangular plate may be explained by a secondary bifurcation - as suggested by Bauer et al. [1] - when "clamped" boundary conditions on the vertical displacement function are assumed. In our analysis we use the singularity theory of mappings in the presence of a symmetry group to analyse the bifurcation equation obtained by the Lyapunov-Schmidt reduction applied to the Von Kármán equations. Noteworthy is the fact that this explanation fails when the assumed boundary conditions are "simply supported".

Mode jumping in the presence of "clamped" boundary conditions was observed experimentally by Stein [9]; "simply supported" boundary conditions are frequently studied but are difficult - if not impossible - to realize physically. Thus, it is important to observe that the qualitative post-buckling behavior depends on which idealization for the boundary conditions one chooses.
\end{abstract}

Mode jumping [9] is perhaps the most noteworthy feature of experimental studies of the post-buckling behavior of plates. As is well known, a rectangular plate can support a number of different buckled configurations; these may be distinguished by their wave number, by which we mean the number of zeroes of the (normal) deflection function along a line parallel to the leading direction. Experiments [9] have shown that the wave number need not remain constant as the load is gradually increased past the buckling load; rather there are special values of the load parameter at which a sudden and violent change in buckling pattern occurs. The new mode typically has a wave number greater than the old mode by unity.

A spring model proposed by Stein [8] offers an attractive explanation of this phenomenon. As observed by Bauer et al. [1] secondary bifurcation often results from splitting a double eigenvalue by perturbation; in the spring model mode

* Research sponsored in part by the U.S. Army Contract DAAG29-75-C-0024 and the N.S.F. Grant MCS77-04148

$\star \star$ Research partially supported by the N.S.F. Grant MCS77-03685 and the Research Foundation of C.U.N.Y. 
jumping could occur [1] when the primary solution branch lost stability through such a secondary bifurcation. Whether or not mode jumping actually occurs depends on the value of certain parameters. In [4] we analyzed the most general form of reduced bifurcation equations for a rectangular plate at a double eigenvalue, consistent with the symmetries of the von Kármán equations (or any other plate theory). It follows from this analysis that the spring model already exhibits (essentially) all possible behavior of the plate, and moreover that whether the plate exhibits mode jumping is determined by two dimensionless parameters which - using the terminology of singularity theory - we call modal parameters. Calculations by Matkowsky and Putnik [6], Chow et al. [2], and Magnus and Poston [5] cast doubt on this explanation of mode jumping. These authors analyzed a simply supported plate governed by the von Kármán equations and (in our language) found that the modal parameter values were such that mode jumping would not occur. However simply supported boundary conditions are hard to achieve experimentally, and in fact Stein [9] suggests that clamped boundary conditions would be the most accurate approximation for the loaded ends. Therefore, in this paper we analyze a von Kármán plate subject to these mixed boundary conditions - clamped at the loaded edges, simply supported at the unloaded edges - and we find that here mode jumping does occur. (For comparison we also consider the case of simply supported boundary conditions on all four sides.) Of course, given the many doubts surrounding the von Kármán equation, the most important result of this paper is perhaps that even the qualitative behavior of a buckled plate may be changed by the choice of boundary conditions.

The reader should be warned that the calculations outlined in Sect. 8 are long and tedious. However, the fact that these computations are made without the use of a computer is a simplification when compared to other work in this area. Moreover, our choice of boundary conditions made this simplification possible and this choice was prompted by physical considerations alone.

\section{The Experiment of Stein and Its Mathematical Idealization}

Let us begin by a description of Stein's [9] apparatus. In Fig. 1 we have indicated part of a large plate divided into 11 panels, each $4.71^{\prime \prime}$ by $25.36^{\prime \prime}$. The division is performed by knife blades located on either side of the plate, as shown in the end view. The knife blades prevent any normal displacement while (in principle) not inhibiting motion in the plane. Actually one is interested in the buckling of just a single panel, but having many, more or less identical, adjacent panels provides an experimentally feasible way of achieving simply supported boundary conditions along the unloaded edges. All measurements were performed on the center panel, which for theoretical purposes is treated as being embedded in an infinite periodic array of such panels.

In the experiment, when the load was first increased beyond the buckling load, the initial configuration of the plate contained 5 buckles. This pattern persisted as the load was gradually increased until a load approximately 1.7 times the buckling load was passed, when the plate jumped suddenly and violently to a new configuration with 6 buckles. Further increases in the load led to jumps to states with 7 and 8 buckles, and eventually to the complete collapse of the plate. These latter jumps occurred after the plate had begun to deform plastically and are not discussed here - we consider only the jump from 5 buckles to 6 . 


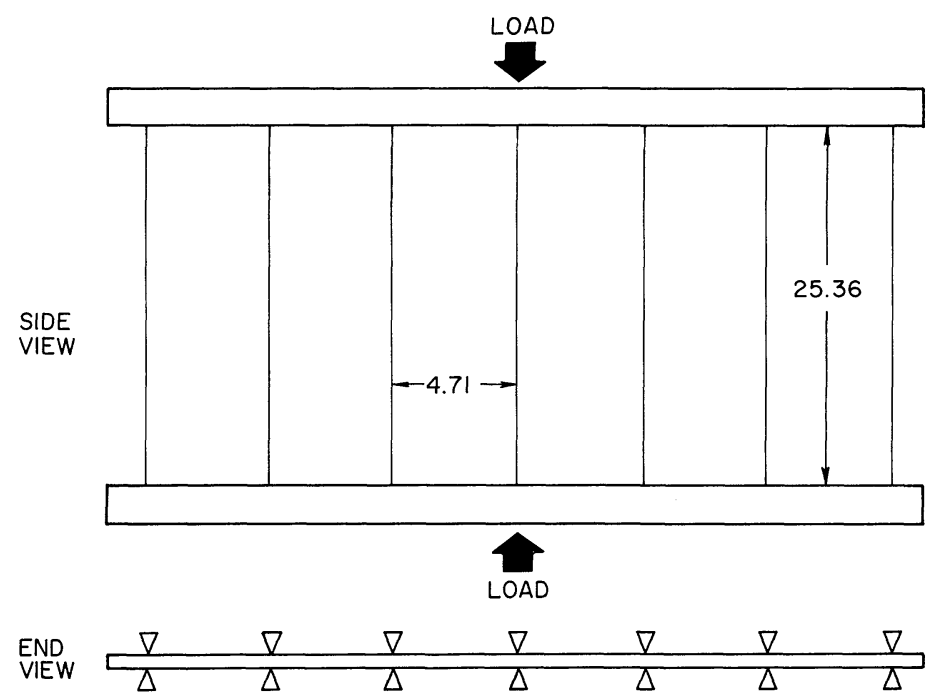

Fig. 1

Fig. 2

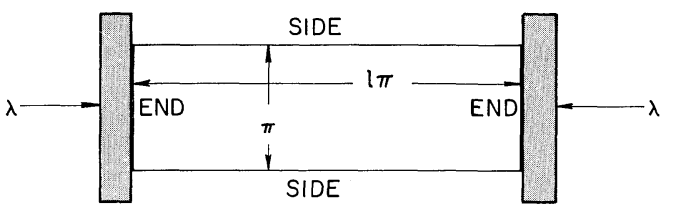

For computational convenience in our theoretical analysis we choose a length scale so that the plate has width $\pi$ and length $l \pi$. Thus the undeformed plate is parameterized by

$$
\Omega=\left\{\left(z_{1}, z_{2}\right): 0 \leqq z_{1} \leqq l \pi, 0 \leqq z_{2} \leqq \pi\right\} .
$$

The plate is subjected to a load $\lambda$ applied to the ends through a rigid yoke as indicated in Fig. 2. The von Kármán equations for $w$, the $z_{3}$-deflection of the plate, and $\phi$, the Airy stress function, are as follows

$$
\begin{aligned}
\Delta^{2} w & =[\phi, w]-\lambda w_{z_{1} z_{1}} \\
\Delta^{2} \phi & =-\frac{1}{2}[w, w] .
\end{aligned}
$$

Here $\Delta^{2}$ is the biharmonic operator in the plane and

$$
[u, v]=u_{z_{1} z_{1}} v_{z_{2} z_{2}}-2 u_{z_{1} z_{2}} v_{z_{1} z_{2}}+u_{z_{2} z_{2}} v_{z_{1} z_{1}}
$$

the subscripts indicating differentiation. We analyze two sets of boundary conditions for $w$, namely

$$
\left.\begin{array}{l}
w=w_{N}=0 \text { on ends (clamped) } \\
w=\Delta w=0 \text { on sides (simply supported) }
\end{array}\right\} .
$$

(The subscript $N$ indicates differentiation in the normal direction.) We are most interested in (1.3i) as it seems to be the best approximation for Stein's experiment (We quote: "The plate was subject ... to 'flat end' loading which results in almost 
complete clamping of the loaded edges."), but we also consider (1.3ii) for the sake of comparison. In both cases above we take

$$
\phi_{N}=(\Delta \phi)_{N}=0
$$

as the boundary condition for $\phi$; this differs somewhat from the usual choice, and we explain our reasons in Sect. 2.

The paper is structured as follows. After discussing the boundary conditions for the von Kármán equations in the next section, we give a variational formulation in Sect. 3. The eigenvalues and eigenfunctions for the linearized von Kármán equations are computed in Sect. 4 along with a description of the relevant symmetries for this problem. In Sect. 5, we use results from Sects. 3 and 4 to compute the Lyapunov-Schmidt reduction to lowest order. The results from singularity theory [4] which form the basis for the rigorous analysis given here are sketched in Sect. 6. The main result is that all small perturbations of the von Kármán equations near a double eigenvalue consistent with the symmetries of Sect. 4 may be described - in an appropriate qualitative sense - by the addition of just one more parameter. As a consequence the whole perturbed bifurcation problem can be given an explicit normal form from which computations about secondary bifurcation may be made. The diagrams showing how mode jumping may be accomplished are also given in this section. The modal parameters for the two choices of boundary conditions are computed in Sects. 7 and 8. Finally, in Sect. 9 we show that the aspect ratio $l$ may be taken to be the one non-modal perturbation parameter.

\section{Boundary Conditions in the von Kármán Equations}

We have discussed above the two choices (1.3) for boundary conditions for $w$ along the loaded ends. The simply supported boundary conditions for $w$ along the sides were chosen for the following reason. The end view of a typical cross section of the plate after buckling is indicated in Fig. 3. Assuming the center panel is embedded in an infinite periodic array, the displacement $w$ is an odd function under reflection across either side of the panel, so

$$
w=w_{N N}=0 \quad \text { on sides. }
$$

On adding a second order tangential derivative to $w_{N N}=0$ we obtain $\Delta w=0$, as taken in (1.3).

Fig. 3

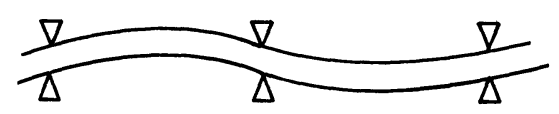

The boundary conditions for the stress function are more subtle, perhaps because $\phi$ is a derived quantity in the theory. In the remainder of this section we indicate explicitly the hypotheses under which (1.4) follows from the equations of continuum mechanics. The reader prepared to accept (1.4) may omit this section without loss of continuity. Most interestingly we found that the boundary conditions (1.4), which we consider most realistic for the experiment, actually simplified the calculations as compared to the more customary choice $\phi=\Delta \phi=0$. 
Consider a three dimensional solid occupying a region $U$ in its undeformed position. For the rectangular plate

$$
U=\left\{0 \leqq z_{1} \leqq \pi l, 0 \leqq z_{2} \leqq \pi,-h \leqq z_{3} \leqq h\right\} .
$$

A deformation of the solid is a map $\Phi: U \rightarrow \mathbb{R}^{3}$. The principle unknowns in continuum mechanics are the three components of the displacement $u=\left(u_{1}, u_{2}, u_{3}\right)$ defined by

$$
\Phi(z)=z+u(z)
$$

where $z=\left(z_{1}, z_{2}, z_{3}\right)$. The strain tensor is defined by

$$
\varepsilon_{i j}=\frac{1}{2}\left(\frac{\partial u_{i}}{\partial z_{j}}+\frac{\partial u_{j}}{\partial z_{i}}+\sum_{l=1}^{3} \frac{\partial z_{l}}{\partial z_{i}} \frac{\partial u_{l}}{\partial z_{j}}\right) .
$$

The stress tensor $\sigma_{i j}$ is determined from the strain by some sort of constitutive relation. For the problem at hand the strains are small and Hooke's law is considered sufficiently accurate. Thus, stress and strain are related linearly by

$$
\varepsilon_{i j}=\frac{1}{E}\left\{(1+v) \sigma_{i j}-v\left(\sum_{l=1}^{3} \sigma_{l l}\right) \delta_{i j}\right\},
$$

where $E$ is Young's modulus, $v$ is Poisson's ratio, and $\delta_{i j}$ is the Kronecker notation. Of course (2.3) may be inverted to give $\sigma$ as a function of $\varepsilon$. Finally the equilibrium equations are

$$
\sum_{j=1}^{3} \frac{\partial \tilde{\sigma}_{i j}}{\partial \tilde{z}_{j}}=0,
$$

where the tilde indicates variables expressed in the deformed coordinates resulting from the transformation $\Phi$. Equation (2.4) represents a system of three equations for the three components of $u$.

The derivation of the von Kármán equations from (2.4) is based on two approximations which, moreover, are intertwined in a subtle way. First, it is assumed that the plate is very thin (i.e., $h \ll 1$ ), and second, it is assumed that deflections are small with the $z_{3}$-deflection $\left(u_{3}\right)$ predominating. This derivation, rather heuristic in character, is carried out in Chap. $\mathrm{V}$ of [10], for example, and yields the PDE

$$
\begin{aligned}
\Delta^{2} w & =[\phi, w] \\
\Delta^{2} \phi & =-\frac{1}{2}[w, w],
\end{aligned}
$$

where $w=u_{3}$. We mention here that the Airy stress function $\phi\left(z_{1}, z_{2}\right)$ is defined by the relations

$$
\phi_{z_{2} z_{2}}=\bar{\sigma}_{11}, \quad \phi_{z_{1 z_{2}}}=-\bar{\sigma}_{12}, \quad \phi_{z_{1 z_{1}}}=\bar{\sigma}_{22},
$$

where the bars indicate averages over the thickness of the plate. For example,

$$
\bar{\sigma}_{11}\left(z_{1}, z_{2}\right)=\frac{1}{2 h} \int_{-h}^{h} \sigma_{11}\left(z_{1}, z_{2}, z_{3}\right) d z_{3} .
$$


Since $\phi$ is defined by second order differential equations we may without loss of generality assume

$$
\phi_{z_{1}}(0,0)=\phi_{z_{2}}(0,0)=0=\int_{\Omega} \phi .
$$

The relations (2.6) and (2.7) provide the basis for the choice of boundary conditions for $\phi$. Below we shall omit the bars in (2.6) - indeed to lowest order in the thickness parameter $h$ all quantities are independent of $z_{3}$ and thus equal their averages.

The approximations indicated above mean that a number of terms in (2.2) are negligibly small; indeed to this level of approximation we have

$$
\begin{aligned}
\varepsilon_{12} & =\frac{1}{2}\left(\frac{\partial u_{1}}{\partial z_{2}}+\frac{\partial u_{2}}{\partial z_{1}}+\frac{\partial u_{3}}{\partial z_{1}} \frac{\partial u_{3}}{\partial z_{2}}\right) \\
\varepsilon_{i i} & =\frac{\partial u_{i}}{\partial z_{i}}+\frac{1}{2}\left(\frac{\partial u_{3}}{\partial z_{i}}\right)^{2} i=1,2
\end{aligned}
$$

We now analyze the boundary conditions for $\phi$, first along the loaded end of the plate given by $z_{1}=0$. Those boundary conditions which seem the most relevant here are

$$
\frac{\partial u_{1}}{\partial z_{2}}=0 \text { and } \quad \sigma_{12}=0 \text { along } z_{1}=0 .
$$

For imagine the experimental configuration of Fig. 4 in which the plate is compressed by some supporting structure. We suppose that the bar in the supporting frame is rigid, so that whatever compression of the plate occurs will be uniform along the edge. This condition is expressed by the equation

$$
\frac{\partial u_{1}}{\partial z_{2}}=0 \quad \text { on } \quad z_{1}=0
$$

The other boundary condition in (2.9) expresses the condition that there is no friction between the plate and the compressing bar, the plate is free to slide along the length of the bar. In the following lemma $\frac{\partial}{\partial N}$ and $\frac{\partial}{\partial T}$ denote normal and tangential derivatives.

Lemma 2.10. The boundary conditions (2.9) imply

$$
\frac{\partial}{\partial T}\left(\frac{\partial \phi}{\partial N}\right)=0 \quad \text { and } \quad \frac{\partial}{\partial N}(\Delta \phi)=0 \quad \text { along } \quad z_{1}=0 \text {. }
$$

Proof. Observe that $u_{3}=0$ along $z_{1}=0$ as $u_{3}=w$ in the von Kármán equations and $w$ vanishes along all edges for both our choices of boundary conditions. Since $\frac{\partial}{\partial z_{2}}$ is a tangential derivative, $\frac{\partial u_{3}}{\partial z_{2}}=0$ on $z_{1}=0$. 
Now by (2.3) $\varepsilon_{12}$ and $\sigma_{12}$ are proportional, and by (2.9) both vanish on $z_{1}=0$. Thus we may use (2.8) to modify the first equation of (2.9) to read (note the interchange of indices)

$$
\frac{\partial u_{2}}{\partial z_{1}}=0 \quad \text { and } \quad \sigma_{12}=0 \quad \text { on } \quad z_{1}=0
$$

On taking the tangential derivative $\frac{\partial}{\partial z_{2}}$ we find

$$
\frac{\partial^{2} u_{2}}{\partial z_{1} \partial z_{2}}=0 \text { and } \quad \sigma_{12}=0 \quad \text { on } \quad z_{1}=0
$$

Now (2.8) implies that

$$
\frac{\partial \varepsilon_{22}}{\partial z_{1}}=\frac{\partial^{2} u_{2}}{\partial z_{1} \partial z_{2}}+\frac{\partial u_{3}}{\partial z_{2}} \frac{\partial^{2} u_{3}}{\partial z_{1} \partial z_{2}} \text {. }
$$

As $\frac{\partial u_{3}}{\partial z_{2}}=0$ on $z_{1}=0$ we replace $(2.13)$ with

$$
\sigma_{12}=0=\frac{\partial \varepsilon_{22}}{\partial z_{1}} \text { on } z_{1}=0 \text {. }
$$

In view of Hooke's law (2.3) we are led to the boundary conditions

$$
\sigma_{12}=0=\frac{\partial}{\partial z_{1}}\left(\sigma_{22}-v \sigma_{11}\right) \text { on } z_{1}=0 .
$$

Note that $\sigma_{3 i}$ is assumed to be zero in the approximations made to derive the von Kármán equations.

Using (2.6) we may write (2.16) in terms of $\phi$ obtaining:

$$
\phi_{z_{1} z_{2}}=0=\phi_{z_{1} z_{1} z_{1}}-v \phi_{z_{1} z_{2} z_{2}} \text { on } z_{1}=0 \text {. }
$$

The first equations of (2.11) and (2.17) coincide. Moreover $\phi_{z_{1} z_{2} z_{2}}=0$ on $z_{1}=0$ since $\frac{\partial}{\partial z_{2}}$ is a tangential derivative, and it follows that

$$
\frac{\partial}{\partial z_{1}}(\Delta \phi)=0 \quad \text { on } \quad z_{1}=0 .
$$

This proves Lemma 2.10 .

We claim that (2.11) is also satisfied along the sides of $\partial \Omega$. Recall from Sect. 1 that the boundary conditions along the sides are manufactured by assuming periodic behavior in the $z_{2}$ variable. This periodicity for the stresses leads to

$$
\sigma_{12}=0, \quad \frac{\partial}{\partial N} \sigma_{11}=0, \text { and } \frac{\partial}{\partial N} \sigma_{22}=0
$$

on the sides as $\sigma_{i i}$ is even with respect to reflection across the lateral boundaries while $\sigma_{12}$ is odd. By (2.6) 
on the sides. On omitting the redundant middle equation we obtain boundary conditions equivalent to (2.11).

Lemma 2.19. Let $\tilde{\phi}\left(z_{1}, z_{2}\right)=\phi\left(z_{1}, z_{2}\right)+\frac{1}{2} \lambda z_{2}^{2}$. Then $\tilde{\phi}$ and $w=u_{3}$ satisfy:

$$
\begin{aligned}
& \Delta^{2} w=-\lambda w_{z_{1} z_{1}}+[\tilde{\phi}, w] \quad \text { in } \Omega \\
& \Delta^{2} \tilde{\phi}=-\frac{1}{2}[w, w]
\end{aligned}
$$

with boundary conditions

$$
\frac{\partial \tilde{\phi}}{\partial N}=0 \quad \text { and } \quad \frac{\partial}{\partial N} \Delta \tilde{\phi}=0 \quad \text { on } \quad \partial \Omega
$$

Note. This is the form for the von Kármán equations which was described in Sect. 1.

Proof. It is trivial to see that $\tilde{\phi}$ satisfies (2.20) as $\phi$ satisfies (2.5). By (2.11)

$$
\frac{\partial}{\partial N}(\Delta \phi)=0 \quad \text { on } \quad \partial \Omega
$$

and $\tilde{\phi}$ satisfies the same boundary condition as it differs from $\phi$ by a quadratic polynomial which is annihilated by the third order operator in (2.22). It follows from (2.11) that $\frac{\partial \phi}{\partial N}$ is constant on each of the four sides of $\partial \Omega$, although the constants could be different on the different sides. With the normalization (2.7) we have

$$
\frac{\partial \phi}{\partial N}=0 \quad \text { on } \quad z_{1}=0 \text { and } z_{2}=0
$$

while

$$
\frac{\partial \phi}{\partial N}=c_{1} \quad \text { on } \quad z_{1}=l \pi \quad \text { and } \quad \frac{\partial \phi}{\partial N}=c_{2} \quad \text { on } \quad z_{2}=\pi .
$$

It remains to determine $c_{1}$ and $c_{2}$. Observe that

$$
\begin{aligned}
& \phi_{z_{1}}(l \pi, 0)-\phi_{z_{1}}(0,0)=\int_{0}^{l \pi} \phi_{z_{1} z_{1}}\left(z_{1}, 0\right) d z_{1} \\
& \phi_{z_{2}}(0, \pi)-\phi_{z_{2}}(0,0)=\int_{0}^{\pi} \phi_{z_{2} z_{2}}\left(0, z_{2}\right) d z_{2} .
\end{aligned}
$$

Applying (2.7) and (2.6) we have

$$
\begin{aligned}
& \phi_{z_{1}}(l \pi, 0)=\int_{0}^{l \pi} \sigma_{22}\left(z_{1}, 0\right) d z_{1}=0 \\
& \phi_{z_{2}}(0, \pi)=\int_{0}^{\pi} \sigma_{11}\left(0, z_{2}\right) d z_{2}=-\lambda,
\end{aligned}
$$


the latter equalities coming from the total compressive force exerted on the plate. Note that the total force - described by the integrals - is given even though its distribution is not. Thus $c_{1}=0$ and $c_{2}=-\lambda$. Equation (2.24) leads to (2.21) on recalling the definition of $\tilde{\phi}$. The proof is complete.

For the remainder of this section we describe - though not in detail - other possible choices for boundary conditions for the von Kármán equations.

If, in Fig. 4., the plate were welded to the compressing bar, the appropriate boundary conditions would be:

$$
\frac{\partial u_{1}}{\partial z_{2}}=0, \quad u_{2}=0 \text { on the ends. }
$$

This is a physically consistent boundary condition which, however, does not seem to be expressible in terms of the stress function $\phi$, at least not without the use of pseudodifferential operators. It also seems less appropriate for Stein's experiment.

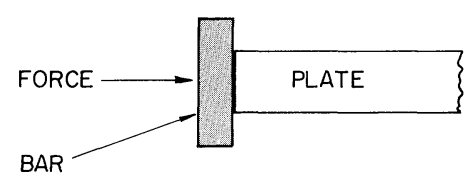

Fig. 4

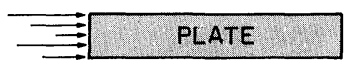

Fig. 5

A different experimental arrangement is depicted in Fig. 5. Here the compressive force is administered by a large number of smaller forces applied locally, each at the control of the experimenter. (By fixing the force one looses control over the displacement.) Idealizing one imagines that the force density is prescribed all along the boundary.

In view of the relation

$$
F_{i}=\sum_{j} \sigma_{i j} n_{j}
$$

this boundary condition leads to the prescription of $\sigma_{11}$ and $\sigma_{12}$ and thus by (2.6) to the prescription of $\phi_{z_{1 z_{2}}}$ and $\phi_{z_{z_{2} z_{2}}}$. On integrating the tangential derivatives, one obtains $\phi$ and $\phi_{N}$ on the boundary. In other words this configuration leads to Dirichlet boundary conditions. Although conceptually possible, experimental realization of these boundary conditions might prove difficult.

A common boundary condition $[2,5,6]$ used for $\phi$ is

$$
\phi=\Delta \phi=0 \quad \text { on } \quad \partial \Omega .
$$

This boundary condition implies that

$$
\phi_{z_{1 z_{1}}}=\phi_{z_{2 z_{2}}}=0 \quad \text { on } \quad \partial \Omega
$$

which is equivalent - by (2.6) - to

$$
\sigma_{11}=0 \text { and } \sigma_{22}=0 \text { on } \partial \Omega \text {. }
$$


As noted above in (2.29) $\sigma_{11}$ is related to the $z_{1}$ component of the external forces and may therefore be prescribed. However, $\sigma_{22}$ is related to the stress transmitted within the plate parallel to the edge. It is a non-trivial question in control theory to decide whether one can achieve an arbitrary profile for $\sigma_{22}$ by operations performed at the boundary of the plate. Certainly prescription of $\sigma_{22}$ is an unphysical boundary condition.

\section{A Variational Formulation}

In this section we show that solutions of the von Kármán equations with boundary conditions (1.3), (1.4) may be characterized as stationary points of the potential

$$
V(w)=\frac{1}{2}\|\Delta w\|^{2}-\frac{\lambda}{2}\left\|w_{z_{1}}\right\|^{2}+\frac{1}{8}\left\|\Delta_{N}^{-1}[w, w]\right\|^{2},
$$

where $\|\cdot\|$ indicates the $L^{2}$ norm and $\Delta_{N}^{-1}$ is the generalized inverse of the Laplacian with Neumann boundary conditions. The domain of $V$ consists of functions in $\mathscr{H}_{2}(\Omega)$ (i.e., two derivatives in the $L^{2}$ sense) which satisfy either

$$
\begin{aligned}
& w=w_{N}=0 \text { on ends, } \quad w=0 \text { on sides, } \\
& w=0 \text { all around }
\end{aligned}
$$

according as (1.3i) or (1.3ii) is desired.

One can easily eliminate $\phi$ from the von Kármán equations with boundary conditions (1.4), regarding $w$ as given one determines $\phi$ by solving

$$
\begin{aligned}
& \Delta^{2} \phi=-\frac{1}{2}[w, w] \text { on } \Omega \\
& \phi_{N}=(\Delta \phi)_{N}=0 \text { on } \partial \Omega \text {. }
\end{aligned}
$$

Indeed (3.3) is a boundary problem associated with $\Delta_{N}^{2}$, the square of the Laplace operator on $L^{2}(\Omega)$ with Neumann boundary conditions, so we may write $\phi=-\frac{1}{2} \Delta_{N}^{-2}[w, w] .\left[\Delta_{N}\right.$ has a one dimensional kernel spanned by the constants. The generalized inverse $\Delta_{N}^{-1}$ is defined by the requirements

$$
\left.\Delta_{N}^{-1} 1=0 \text { and } \int \Delta_{N}^{-1} f d x=0 \text { for } f \in L^{2}(\Omega) .\right]
$$

The equation for $w$ may therefore be written

$$
\Delta^{2} w=-\lambda w_{z_{1} z_{1}}-\frac{1}{2}\left[\Delta_{N}^{-2}[w, w], w\right] \text {. }
$$

Suppose $w$ is a stationary point of $V$ with either (3.2i) or (3.2ii) defining the domain. Regardless of boundary conditions we certainly have $d V_{w} \cdot \zeta=0$ for all test functions $\zeta \in \mathscr{D}(\Omega)$. A simple calculation shows that

$$
d V_{w} \cdot \zeta=(\Delta w, \Delta \zeta)-\lambda\left(w_{z_{1}}, \zeta_{z_{1}}\right)+\frac{1}{2}\left(\Delta_{N}^{-1}[w, w], \Delta_{N}^{-1}[w, \zeta]\right)
$$

where $(u, v)=\int_{\Omega} u v d z$ denotes the $L^{2}$ inner product. [Note that the third term in (3.1) is homogeneous of degree 4 and differentiation brings out a factor 4 .] We now 
integrate by parts in the sense of distributions in (3.5) to obtain

$$
d V_{w} \cdot \zeta=\left(\Delta^{2} w+\lambda w_{z_{1} z_{1}}+\frac{1}{2}\left[\Delta_{N}^{-2}[w, w], w\right], \zeta\right) .
$$

In the case of the first two terms the integration by parts is justified because $\zeta \in \mathscr{D}(\Omega)$, while for the third it may be justified by shifting the bounded, self-adjoint operator $\Delta_{N}^{-1}$ to the other factor and then appealing to the following lemma with $f=\Delta_{N}^{-2}[w, w]$. Since (3.6) must vanish for all $\zeta \in \mathscr{D}(\Omega)$, we conclude that (3.4) is satisfied in the weak sense. However (3.4) is an elliptic equation, and it follows that the equation is also satisfied in the classical sense.

Lemma 3.7. If $f, w, \zeta \in \mathscr{H}_{2}(\Omega)$ and if $w, \zeta$ vanish on $\partial \Omega$, then

$$
\int_{\Omega} f[w, \zeta] d z=\int_{\Omega}[f, w] \zeta d z
$$

Remark 3.9. It may be seen as follows that the integrals in (3.8) are meaningful. The bracket operation involves second derivatives of its arguments, which for $\mathscr{H}_{2}$ functions contributes products of $L^{2}$ functions. On the other hand, by Sobolev's lemma in two dimension, an $\mathscr{H}_{2}$ functions is continuous and therefore bounded. Thus the triple products in (3.8) are integrable.

Proof. First suppose that $f, w, \zeta$ are smooth. Ignoring the possible boundary terms in an integration by parts we find that

$$
\begin{aligned}
\int_{\Omega} f[w, \zeta] d z & =\int_{\Omega}\left\{\frac{\partial^{2}}{\partial z_{2}^{2}}\left(f w_{z_{1} z_{1}}\right)-2 \frac{\partial^{2}}{\partial z_{1} \partial z_{2}}\left(f w_{z_{1} z_{2}}\right)+\frac{\partial^{2}}{\partial z_{1}^{2}}\left(f w_{z_{2} z_{2}}\right)\right\} \zeta d z \\
& =\int_{\Omega}[f, w] \zeta d z,
\end{aligned}
$$

the latter equality being the result of cancellation. It remains to show that the boundary terms vanish. In the first integration by parts the boundary term is

$$
\int_{\Omega} f\langle Q, N\rangle d S
$$

where the two component vector $Q$ is given by

$$
Q=\left(w_{z_{2} z_{2}} \zeta_{z_{1}}-w_{z_{1} z_{2}} \zeta_{z_{2}}, w_{z_{1} z_{1}} \zeta_{z_{2}}-w_{z_{1} z_{2}} \zeta_{z_{1}}\right)
$$

On a portion of the boundary where $N=(0, \pm 1)$ both terms in the second component of $Q$ vanish, since $w_{z_{1} z_{1}}$ and $\zeta_{z_{1}}$ are tangential derivatives and both $w$ and $\zeta$ vanish along the boundary. Similarly for the case $N=( \pm 1,0)$. Thus the first integration by parts contributes no boundary term. In the second integration by parts, the boundary integral contains a factor of $\zeta$ and therefore vanishes.

We have verified (3.8) when $f, w, \zeta$ are $C^{\infty}$. In the general case consider sequences $f_{n}, w_{n}, \zeta_{n}$ of smooth functions which converge to the appropriate functions in the $\mathscr{H}_{2}$ norm. We claim that the integrals in (3.8) are continuous with respect to this convergence; indeed this follows from the observations of Remark 3.9. Thus (3.8) extends to the limit functions, and the proof is complete.

We now consider the role of boundary conditions. In case (3.2ii) the domain of $V$ is

$$
X=\left\{w \in \mathscr{H}_{2}(\Omega): w=0 \text { on } \partial \Omega\right\} .
$$


Suppose $w \in X$ is a stationary point of $V$. Since $w \in X$ the first boundary condition in (1.3ii) is satisfied automatically. To verify the second we again integrate by parts in the relation $d V_{w} \cdot \zeta=0$ for $\zeta \in X$. Keeping track of the boundary contribution of the first term in (3.5) we find

$$
\begin{aligned}
d V_{w} \cdot \zeta= & \left(\Delta^{2} w+\lambda w_{z_{1 z_{1}}}+\frac{1}{2}\left[\Delta_{N}^{-2}[w, w], w\right], \zeta\right) \\
& +\int_{\partial \Omega}\left\{\Delta w \frac{\partial \zeta}{\partial N}-\frac{\partial}{\partial N}(\Delta w) \zeta\right\} d S .
\end{aligned}
$$

Since Eq. (3.4) is satisfied, the inner product in (3.11) vanishes. Likewise the second term in the boundary integral contributes nothing since $\zeta$, which vanishes on $\partial \Omega$, is a factor. Thus we have shown that

$$
\int_{\partial \Omega} \Delta w \frac{\partial \zeta}{\partial N} d S=0 \text { for all } \zeta \in X
$$

from which it follows that $\Delta w$ vanishes on $\partial \Omega$, as desired.

The consideration of the case (3.2i) is no more difficult. Along the ends of $\partial \Omega$ the boundary conditions $w=w_{N}=0$ are satisfied automatically by the choice of the domain of $V$, while along the sides the boundary conditions may be verified as above.

\section{The Linearized Problem}

The principal result of this section concerns the linearized version of (3.6) with clamped boundary conditions, namely

$$
\begin{aligned}
& \Delta^{2} w+\lambda w_{z_{1} z_{1}}=0 \text { in } \Omega \\
& w=w_{N}=0 \text { on ends } \\
& w=\Delta w=0 \text { on sides. }
\end{aligned}
$$

We show that the smallest eigenvalue of (4.1) is double if and only if $l=\sqrt{k(k+2)}$ for some positive integer $k$ and that, in this case, the two eigenfunctions are given by (4.14). For completeness we also derive the known result that with simply supported boundary conditions the smallest eigenvalue is double when $l=\sqrt{k(k+1)}$, the eigenfunctions being given by (4.20).

Letting $w=f\left(z_{1}\right) g\left(z_{2}\right)$ one obtains

$$
\frac{f^{[4]}+f^{\prime \prime}}{f}+2 \frac{f^{\prime \prime}}{f} \frac{g^{\prime \prime}}{g}+\frac{g^{[4]}}{g}=0
$$

with boundary conditions

$$
\begin{aligned}
& f(0)=f(l \pi)=f^{\prime}(0)=f^{\prime}(l \pi)=0, \\
& g(0)=g(\pi)=g^{\prime \prime}(0)=g^{\prime \prime}(\pi)=0 .
\end{aligned}
$$

We may satisfy $(4.3 \mathrm{~b})$ by choosing

$$
g\left(z_{2}\right)=\sin \left(m z_{2}\right)
$$


for some positive integer $m$. Moreover the choice $m=1$ leads to the smallest value of $\lambda$ in the bifurcation calculation below, so we only consider this case. Thus

$$
f^{[4]}+(\lambda-2) f^{\prime \prime}+f=0 .
$$

Observe that when $\lambda \leqq 4$ the solutions to (4.5) have a non-oscillatory character and it is impossible to satisfy the boundary conditions (4.3). For $\lambda>4$ the solutions have the form

$$
A \cos \left(a z_{1}\right)+B \sin \left(a z_{1}\right)+C \cos \left(b z_{1}\right)+D \sin \left(b z_{1}\right),
$$

where

$$
a=\sqrt{L-M}, \quad b=\sqrt{L+M}, \quad L=\frac{\lambda}{2}-1, \quad M=\sqrt{\frac{\lambda^{2}}{4}-\lambda .}
$$

The boundary conditions $f(0)=f^{\prime}(0)=0$ imply that $f$ is a linear combination of

$$
\phi=b \sin \left(a z_{1}\right)-a \sin \left(b z_{1}\right) \text { and } \quad \psi=\cos \left(a z_{1}\right)-\cos \left(b z_{1}\right) .
$$

In general it is somewhat tricky to find conditions for when $A \phi+B \psi$ is an eigenfunction. However, we are interested in the case when the first eigenvalue is double so we assume that both $\phi$ and $\psi$ are eigenfunctions. In that case

$$
\phi(l \pi)=\psi(l \pi)=\psi^{\prime}(l \pi)=0
$$

must be satisfied. Note that $\phi^{\prime}=a b \psi$ so that $\phi^{\prime}(l \pi)=0$ is automatic.

Equation (4.9) imply

$$
a=\frac{k}{l}, \quad b=\frac{(k+2 n)}{l},
$$

where $k$ and $n$ are positive integers. From (4.7) we obtain

$$
a^{2}+b^{2}=\lambda-2, \quad b^{2}-a^{2}=\sqrt{\lambda^{2}-4 \lambda} .
$$

Elimination of $\lambda$ yields

$$
a b=1 .
$$

Thus

$$
l=\sqrt{k(k+2 n)} .
$$

Since we are interested only in the first bifurcation we assume that $n=1$ in (4.13). The eigenfunctions for this problem are

$$
\begin{aligned}
& w_{1}=\left(\frac{k+2}{k} \sin \left(\frac{k z_{1}}{l}\right)-\sin \left(\frac{(\mathrm{k}+2) \mathrm{z}_{1}}{l}\right)\right) \sin \left(z_{2}\right) \\
& w_{2}=\left(\cos \left(\frac{k z_{1}}{l}\right)-\cos \left(\frac{(k+2) z_{1}}{l}\right)\right) \sin \left(z_{2}\right) .
\end{aligned}
$$

Next we compute the eigenfunctions for the von Kármán equations with simply supported boundary conditions. We wish to find solutions to (4.2) where 
both $f$ and $g$ satisfy (4.3b). Again only solutions $f\left(z_{1}\right)=\sin \left(a z_{1}\right)$ are consistent with these boundary conditions. Similarly for $g$. Thus the eigenfunctions have the form

$$
\sin \left(\frac{k}{l} z_{1}\right) \sin \left(m z_{2}\right)
$$

with the associated eigenvalue being

$$
\lambda=\frac{l^{2}}{k^{2}}\left(\frac{k^{2}}{l^{2}}+m^{2}\right)^{2} .
$$

As $\lambda$ is increasing in $m$ the first eigenvalue for a given $l$ is when $m=1$ so (4.16) implies

$$
\lambda_{k}=\left(\frac{k}{l}+\frac{l}{k}\right)^{2} .
$$

Note that $\lambda_{k}$ has a unique minimum at $k=l$ when $k$ is viewed as a continuous parameter. Thus the only way for the first eigenvalue to be a double eigenvalue is for

$$
\lambda_{k}=\lambda_{k+1}
$$

with $k<l<k+1$. This happens precisely when

$$
l=\sqrt{k(k+1)} .
$$

Of course the two eigenfunctions are

$$
\begin{aligned}
& w_{1}=\sin \left(\frac{k}{l} z_{1}\right) \sin \left(z_{2}\right) \\
& w_{2}=\sin \left(\frac{k+1}{l} z_{1}\right) \sin \left(z_{2}\right) .
\end{aligned}
$$

We end this section with a discussion of symmetry for the eigenfunctions given in (4.14) and (4.20). There are three obvious symmetries present in the von Kármán equations for a rectangular plate, one of which acts trivially. First, buckling up and buckling down have the same potential energy [see (3.1)]. So this induces a symmetry on the eigenspaces which is given by

$$
\left(w_{1}, w_{2}\right) \rightarrow\left(-w_{1},-w_{2}\right) .
$$

Second the equations are invariant under the mapping

$$
z_{1} \rightarrow l \pi-z_{1}
$$

This also induces an action on the $(\phi, \psi)$ eigenspace $W$. For both (4.14) and (4.20) the action is given by

$$
(\phi, \psi) \rightarrow(-1)^{k+1}(\phi,-\psi) .
$$

The equations are also invariant under the reflection $z_{2} \rightarrow \pi-z_{2}$, but all eigenfunctions in question are invariant under this transformation and no new information is obtained. 
Thus we may assume, by a reordering of $w_{1}$ and $w_{2}$ when $k$ is even, that there is a $Z_{2} \oplus Z_{2}$ group action on $W$ where the first $Z_{2}$ component acts by (4.21) and the second by

$$
\left(w_{1}, w_{2}\right) \rightarrow\left(w_{1},-w_{2}\right) \text {. }
$$

\section{The Lyapunov-Schmidt Reduction}

Proposition 5.15 constitutes the main result of the present section. This proposition gives the bifurcation equations to lowest order after the LyapunovSchmidt reduction has been performed. Note that the constants $a, b, c, \alpha$, and $\beta$ in (5.17) have been defined previously in (5.13).

In Sect. 3 we showed that the von Kármán equations have a variational formulation

$$
d V=0
$$

where $V: X \times \mathbb{R} \rightarrow \mathbb{R}$ and $X$ is the Banach Space (3.10). We may write (5.1) as

$$
\Phi(w, \lambda)=0
$$

where $\Phi: X \times \mathbb{R} \rightarrow X^{*}$ and

$$
(\Phi(w, \lambda), \zeta)=(d V)_{(w, \lambda)}(\zeta)
$$

The calculations of Sect. 4 show that for certain specified lengths $l$ of the plate the smallest eigenvalue $\lambda_{0}$ is a double one; thus in such cases

$$
\operatorname{dim} \operatorname{ker} d \Phi\left(\cdot, \lambda_{0}\right)=2 .
$$

It is also the case that the dimension of coker $d \Phi\left(0, \lambda_{0}\right)=X^{*} /$ range $d \Phi\left(\cdot, \lambda_{0}\right)$ is two. The Lyapunov-Schmidt procedure is a way, using the Implicit Function Theorem, of obtaining a mapping

$$
G: \mathbb{R}^{2} \times \mathbb{R} \rightarrow \mathbb{R}^{2}
$$

such that $G(x, \lambda)=0$ parameterizes the solutions to (5.2). Here the domain $\mathbb{R}^{2}$ is the kernel and the range $\mathbb{R}^{2}$ is the cokernel of $d \Phi\left(\cdot, \lambda_{0}\right)$.

The procedure is simple; let $Q$ be a complementary space to $K=\operatorname{Ker} d \Phi$ and let $E: X^{*} \rightarrow \operatorname{range} d \Phi$ be a projection with kernel $K$. The Implicit Function Theorem guarantees the existence of a mapping

$$
W: K \times \mathbb{R} \rightarrow Q
$$

such that

$$
E \cdot \Phi(k+W(k, \lambda), \lambda) \equiv 0,
$$

where $k \in K$. Let

$$
G(k, \lambda)=(\mathrm{id}-E) \cdot \Phi(k+W(k, \lambda), \lambda) .
$$

This $G$ is the mapping described in (5.5). As $W$ is only defined implicitly the explicit computation of $G$ is impossible. However we shall show that to the lowest nonvanishing order, $W$ drops out of this computation. 
Let $(x, y)$ be the linear coordinates on $K$ given by $w_{1}$ and $w_{2}$ [thus the coordinates of $x w_{1}+y w_{2}$ are $(x, y)$ where $w_{1}$ and $w_{2}$ are the eigenfunctions obtained in Sect. 4]. In these coordinates (5.8) may be rewritten

$$
G(x, y, \lambda)=\left(\left(\Phi\left(x w_{1}+y w_{2}+W\right), w_{1}\right),\left(\Phi\left(x w_{1}+y w_{2}+W\right), w_{2}\right)\right) .
$$

If we define

$$
\tilde{V}(x, y, \lambda)=V\left(x w_{1}+y w_{2}+W(x, y, \lambda), \lambda\right)
$$

then

$$
G(x, y, \lambda)=\left(\frac{\partial \tilde{V}}{\partial x}, \frac{\partial \tilde{V}}{\partial y}\right) .
$$

We claim first that the Taylor expansion of $\tilde{V}$ has the form

$$
\tilde{V}(x, y, \lambda)=a x^{4}+b x^{2} y^{2}+c y^{4}-\alpha \lambda x^{2}-\beta \lambda y^{2}+\ldots,
$$

where the dots indicate higher order terms in $x, y, \lambda$. Note that here $\lambda$ is assumed to vary with $\lambda_{0}$ as origin. We further claim that through terms of the order retained (5.11) $\tilde{V}$ coincides with

$$
\tilde{V}(x, y, \lambda)=V\left(x w_{1}+y w_{2}, \lambda\right) \text {, }
$$

from which $W$ has been omitted entirely. This latter claim follows from the fact that at $x=y=0, \lambda=\lambda_{0}$

$$
W=W_{x}=W_{y}=0 .
$$

In conjunction with (3.1), (5.12) leads to the following explicit formulas for the coefficients in (5.11):

$$
\begin{aligned}
& a=\frac{1}{8}\left\|\Delta_{N}^{-1}\left[w_{1}, w_{1}\right]\right\|^{2} \\
& b=\frac{1}{4}\left[2\left\|\Delta_{N}^{-1}\left[w_{1}, w_{2}\right]\right\|^{2}+\left(\Delta_{N}^{-1}\left[w_{1}, w_{1}\right], \Delta_{N}^{-1}\left[w_{2}, w_{2}\right]\right)\right] \\
& c=\frac{1}{8}\left\|\Delta_{N}^{-1}\left[w_{2}, w_{2}\right]\right\|^{2} \\
& \alpha=\left\|w_{1, z_{1}}\right\|^{2} \\
& \beta=\left\|w_{2, z_{1}}\right\|^{2},
\end{aligned}
$$

where $\Delta_{N}^{-1}$ is the generalized inverse described in Sect. 3 .

We prove the first claim as follows. Observe that the reduced potential function is invariant under the action of $Z_{2} \oplus Z_{2}$ on $K$ described at the end of Sect. 4; that is,

$$
\tilde{V}(x, y)=\tilde{V}(-x, y)=\tilde{V}(x,-y) .
$$

This fact is guaranteed as the full potential function $V$ also satisfies this invariance property [7]. As a result the only possible non-zero terms in the Taylor expansion of $V$ are ones which are even in $x$ and $y$ separately. Thus the form of (5.12) is the most general possible at a double eigenvalue considering the action of this particular symmetry group. Of course this observation could have been obtained by direct calculation! 
Proposition 5.15. The reduced form of the von Kármán equations may be given the form

$$
G(x, y, \lambda)=\left(x^{3}+\mu x y^{2}-\lambda x, v x^{2} y+y^{3}-\lambda y\right)+\ldots,
$$

where

$$
\begin{aligned}
& \mu=\frac{b}{2 c} \frac{\beta}{\alpha} \\
& v=\frac{b}{2 a} \frac{\alpha}{\beta} .
\end{aligned}
$$

Remark 5.18. For reasons which will be discussed in the next section, $\mu$ and $v$ are called modal parameters. They determine the qualitative nature of the actual bifurcation which occurs.

Proof. The preceding discussion shows that

$$
G(x, y, \lambda)=\left(4 a x^{3}+2 b x y^{2}-2 \alpha \lambda x, 2 b x^{2} y+4 c y^{3}-2 \beta \lambda y\right)+\ldots .
$$

Note that appropriate scaling of (5.19) specifically let

$$
x=\sqrt{\frac{\alpha}{2 a}} \bar{x} \text { and } y=\sqrt{\frac{\beta}{2 c}} \bar{y}
$$

and divide the first coordinate of the result by $\alpha \sqrt{\frac{2 \alpha}{a}}$ and the second coordinate by $\beta \sqrt{\frac{2 \beta}{c}}$ leads to (5.16). Note that $a, c, \alpha$, and $\beta$ are all positive so the square roots are real. Of course multiplying each coordinate by a constant and scaling does not change the qualitative nature of the bifurcation diagram $G=0$. This will be formalized in the next section. We will also discuss why the higher order terms in $G$ may be neglected.

\section{Results from Singularity Theory}

What we describe here are results developed in $[3,4]$ for bifurcation problems with symmetry which are applicable to the problem at hand. By a bifurcation problem we mean an equation

$$
G(x, \lambda)=0
$$

where $G: \mathbb{R}^{n} \times \mathbb{R} \rightarrow \mathbb{R}^{n}$ is the germ of a mapping defined near the origin. Let $\Gamma$ be a subgroup of the orthogonal group $O(n)$. A bifurcation problem with symmetry group $\Gamma$ is a bifurcation problem $G$ satisfying

$$
G(\gamma x, \lambda)=\gamma G(x, \lambda)
$$


The preceding analysis of the von Kármán equations yields an example, namely,

$$
G(x, y, \lambda)=\left(x^{3}+\mu x y^{2}-\lambda x, v x^{2} y+y^{3}-\lambda y\right),
$$

where the symmetry group $\Gamma$ is $Z_{2} \oplus Z_{2}$.

Two such bifurcation problems $G$ and $H$ are called contact equivalent if

$$
G(x, \lambda)=T(x, \lambda) \cdot H(X(x, \lambda), \Lambda(\lambda))
$$

where $\frac{\partial \Delta}{\partial \lambda}(0)>0, \operatorname{det}\left(d_{x} X\right)(0)>0$, and for each $(x, \lambda) T(x, \lambda)$ is an invertible $n \times n$ matrix. The problems are $\Gamma$-equivalent if the associated contact equivalence preserves the symmetry group; that is,

$$
\begin{aligned}
& X(\gamma x, \lambda)=\gamma X(x, \lambda) \\
& T(\gamma x, \lambda)=\gamma T(x, \lambda) \gamma^{-1} .
\end{aligned}
$$

The scalings used at the end of Sect. 5 are specific examples of $\Gamma$-equivalences.

Note. No two bifurcation problems of the form (6.3) are $\Gamma$-equivalent unless the corresponding modal parameters $\mu$ and $v$ are identical. Basically this means that no further scaling is possible.

Definition 6.6. A bifurcation problem (6.3) is non-degenerate if $\mu \neq 1, \nu \neq 1$, and $\mu \nu \neq 1$.

One should see $[4, \S 4]$ for a more motivated description of non-degeneracy. We will show that the bifurcation problems obtained from the von Kármán equations are non-degenerate. For now we describe some results.

Proposition 6.7. Let $G$ be a non-degenerate bifurcation problem of type (6.3). Then a bifurcation problem with symmetry group $\Gamma=Z_{2} \oplus Z_{2}$ of the form $G$ plus higher order terms is $\Gamma$-equivalent to $G$.

Proof. Lemma 4.13 of [4].

This proposition justifies the neglect of the higher order terms in the Lyapunov-Schmidt reduction performed in Sect. 5.

Fig. 6

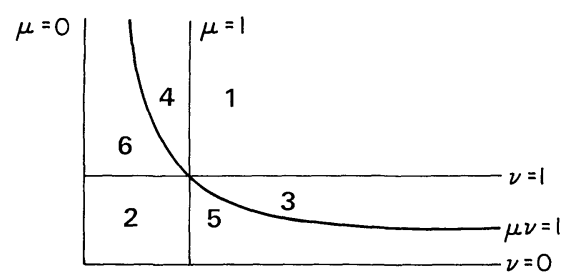

We now analyze the kinds of bifurcation diagrams associated to nondegenerate bifurcation problems. Note that the degeneracies $\mu=1, v=1$, and $\mu \nu=1$ break the first quadrant of the $\mu \nu$-plane into six regions shown in Fig. 6. As shown in [4], if one chooses two pairs $\left(\mu_{1}, v_{1}\right)$ and $\left(\mu_{2}, v_{2}\right)$ in the same region of the $\mu v-$ plane than the bifurcation diagrams obtained by setting (6.3) equal to zero may be 
mapped one onto the other by a homeomorphism of the form $(X(x, y, \lambda), Y(x, y, \lambda)$, $\Lambda(\lambda)$ ). So all diagrams associated to one of the six given regions are topologically equivalent even though there are differential obstructions to contact equivalence (namely, the modal parameters). For all intents and purposes in applications, these regions yield equivalent bifurcation problems. Moreover, interchanging $x$ and $y$ shows that the diagrams in regions 3 and 4 as well as those in regions 5 and 6 are essentially contact equivalent, the equivalence being orientation reversing. With the boundary conditions that we have chosen to analyse, the von Kármán equations always yield $\mu$ greater than one. So we restrict our attention to regions 1 , 3 , and 5. Much of what has been stated here is implicit, though not proved rigorously, in the work of Bauer et al. [1].

A consequence of the unfolding theorem - which is the most powerful theorem in singularity theory - is that one can classify all small perturbations of nondegenerate problems (6.3) up to $\Gamma$-equivalence by the addition of just one new parameter; that is, in addition to the modal parameters discussed above.

Theorem 6.8. Let $H(x, y, \lambda)$ be a bifurcation problem with symmetry group $\Gamma$ $=Z_{2} \oplus Z_{2}$. Suppose that $H$ is a small perturbation of a non-degenerate problem (6.3) with modal parameters $\mu_{0}$ and $v_{0}$. Then $H$ is $\Gamma$-equivalent to

$$
F(x, y, \lambda, \sigma)=\left(x^{3}+\mu x y^{2}-\lambda x, v x^{2} y+y^{3}-(\lambda+\sigma) y\right),
$$

where $(\mu, v, \sigma)$ is near $\left(\mu_{0}, v_{0}, 0\right)$.

Proof. See (4.4) of [4].

The role of $\sigma$, as will be seen, is to split the double eigenvalues into simple eigenvalues. As such it is not surprising that it may be identified with $l$, the length of the plate, which accomplishes the same task for the von Kármán equations. This will be proved in Sect. 9.

The qualitative nature of the bifurcation diagrams associated to (6.9) when $(\mu, v)$ are in regions 1, 3 and 5 are shown in Figs. 7-9 for $\sigma<0$ and $\sigma>0$. Branches which are stable and unstable are indicated by $s$ and $u$ in these figures. The important observation is that when $\sigma<0$, quasi-static variation of $\lambda$ will produce no transition between modes in region (1), smooth transition between branches (or modes) in region (5), and a necessity for mode jumping in region (3). The major point of this paper is that different boundary conditions for the von Kármán equations yield bifurcation problems (6.3) with modal parameters in different regions of the $\mu \nu$-plane. In particular, as will be shown, simply supported boundary conditions yield modal parameters in region 1 while clamped conditions yield modal parameters in region 3 .

To explain the derivation of Figs. 7 through 9, note that setting (6.9) equal to zero yields the equations

$$
\begin{aligned}
& x=0 ; \quad y=0, \\
& y=0 ; \quad x^{2}=\lambda, \\
& x=0 ; \quad y^{2}=\lambda+\sigma, \\
& (v-1) x^{2}+(1-\mu) y^{2}=\sigma ; \quad x^{2}+\mu y^{2}=\lambda .
\end{aligned}
$$



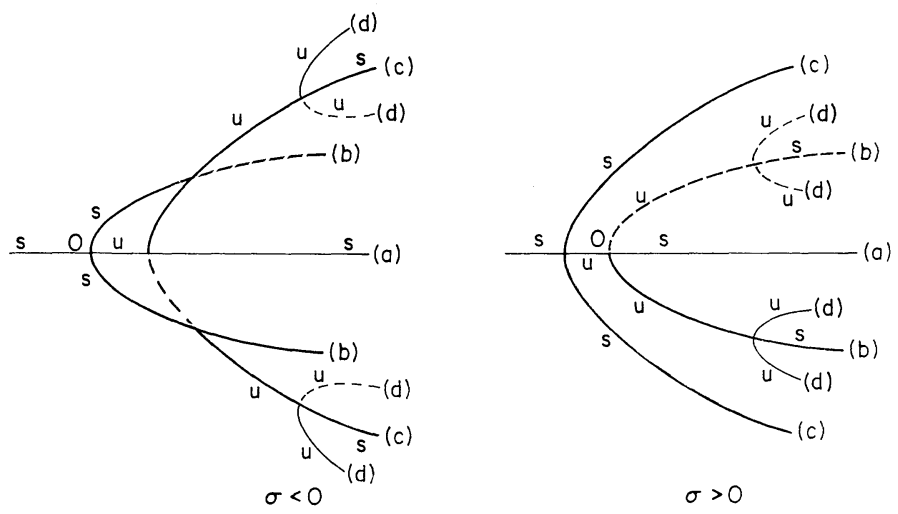

Fig. 7 REGION $1 ; \nu>1$
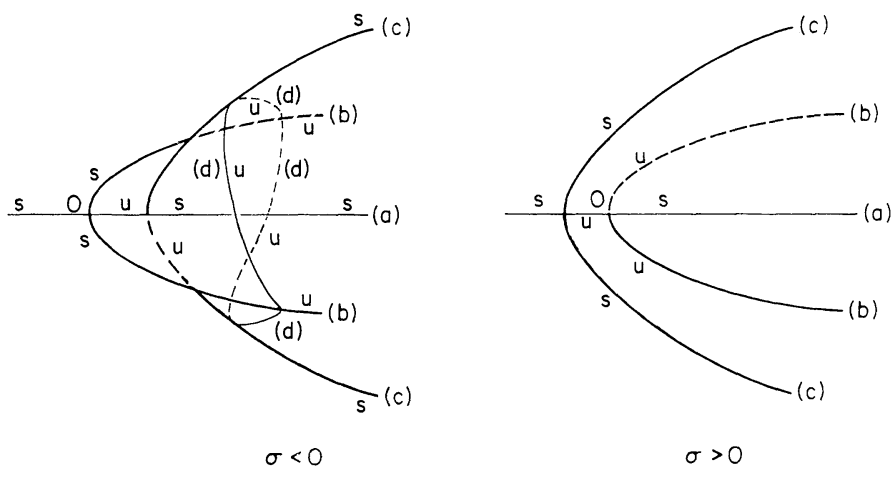

Fig. 8 REGION $3 ; \nu<1, \mu \nu>1$
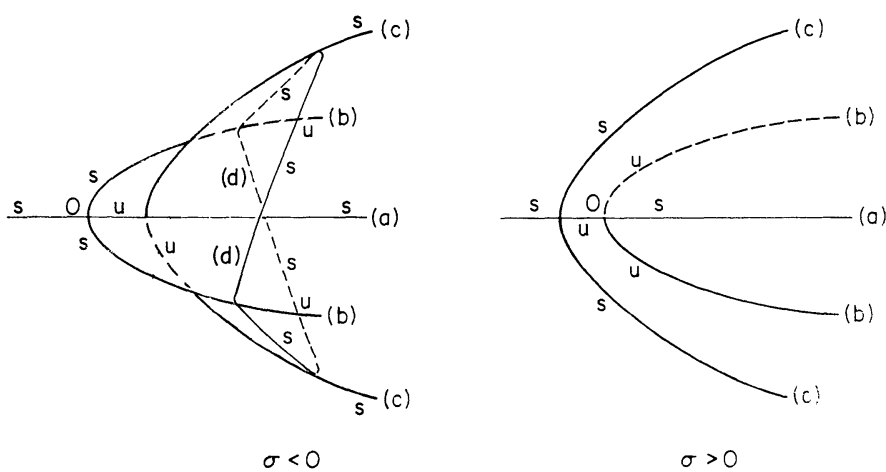

Fig. 9 REGION $5 ; \nu<1, \mu \nu<1$ 
The first three equations have real solution branches in all cases, while the last equation has no real solutions when $\sigma>0$ and $v<1$. Moreover $(6.10 \mathrm{~d})$ may be placed on the figures by noting where the secondary bifurcations occur when branch $(6.10 \mathrm{~d})$ intersects branches $(6.10 \mathrm{~b})$ and $(6.10 \mathrm{c})$. In particular, secondary bifurcations which occur along branch $(6.10 \mathrm{~b})$ occur at

$$
\lambda_{b}=\sigma /(v-1) \text {. }
$$

\section{Calculation of Modal Parameters for the Simply Supported Plate}

Recall that $\Delta_{N}$ is the Laplacian with Neumann boundary conditions. The eigenfunctions of $\Delta_{N}$ are given by

$$
\cos \left(\frac{m}{l} z_{1}\right) \cos \left(n z_{2}\right)
$$

with eigenvalues

$$
-\left(\frac{m^{2}}{l^{2}}+n^{2}\right) \text {. }
$$

In order to compute the modal parameters for the simply supported plate one must compute $a, b, c$ in (5.13). Thus one needs :

$$
A=\Delta_{N}^{-1}\left[w_{1}, w_{1}\right] ; \quad B=\Delta_{N}^{-1}\left[w_{1}, w_{2}\right] ; \quad C=\Delta_{N}^{-1}\left[w_{2}, w_{2}\right],
$$

where $\Delta_{N}^{-1}$ is the generalized inverse of $\Delta_{N}$ defined in Sect. 3. This is easy if one can write $\left[w_{i}, w_{j}\right]$ in a double Fourier cosine series with terms given by (7.1). A computation, involving the trigonometric identities

$$
\begin{aligned}
& 2 \sin u \sin v=\cos (u-v)-\cos (u+v) \\
& 2 \cos u \cos v=\cos (u-v)+\cos (u+v) \\
& 2 \cos ^{2} u=1+\cos (2 u) \\
& 2 \sin ^{2} u=1-\sin (2 u),
\end{aligned}
$$

and the eigenfunctions (4.20) yields

$$
\begin{aligned}
A= & \frac{1}{4}\left[\cos \left(\frac{2 k}{l} z_{1}\right)+\frac{k^{2}}{l^{2}} \cos \left(2 z_{2}\right)\right] \\
B= & \frac{1}{4}\left[\cos \left(\frac{2 k+1}{l} z_{1}\right)-\cos \left(\frac{z_{1}}{l}\right)+\cos \left(\frac{z_{1}}{l}\right) \cos \left(2 z_{2}\right)\right. \\
& \left.-\frac{1}{2(2 k+1)^{2}-1} \cos \left(\frac{2 k+1}{l} z_{1}\right) \cos \left(2 z_{2}\right)\right] \\
C= & \frac{1}{4}\left[\cos \left(\frac{2 k+2}{l} z_{1}\right)+\frac{(k+1)^{2}}{l^{2}} \cos \left(2 z_{2}\right)\right] .
\end{aligned}
$$


Next compute from (5.13) and (7.5) that

$$
\begin{aligned}
& a=\frac{1}{256} \frac{\left(k^{2}+(k+1)^{2}\right)}{(k+1)^{2}} \\
& c=\frac{1}{256} \frac{k^{2}+(k+1)^{2}}{k^{2}} \\
& b=\frac{1}{128}\left[6+\frac{1}{\left(2(2 k+1)^{2}+1\right)^{2}}\right] \\
& \alpha=\frac{k^{2}}{4 l^{2}} \\
& \beta=\frac{(k+1)^{2}}{4 l^{2}} .
\end{aligned}
$$

Now compute using (5.17) that

$$
\begin{aligned}
& \mu=\frac{(k+1)^{2}}{k^{2}+(k+1)^{2}}\left[6+\frac{1}{\left(2(2 k+1)^{2}+1\right)^{2}}\right]>3 \\
& \nu=\frac{k^{2}}{k^{2}+(k+1)^{2}}\left[6+\frac{1}{\left(2(2 k+1)^{2}+1\right)^{2}}\right]>1 .
\end{aligned}
$$

It follows that for any $k$, the bifurcation diagram is qualitatively similar to a problem in region 1 of Fig. 6 when simply supported boundary conditions are assumed.

\section{Calculation of Modal Parameters for the Clamped Plate}

The first step in these computations is to compute $A, B$, and $C$ from Sect. 7 with the eigenfunctions associated to the clamped plate:

$$
w_{1}=\phi\left(z_{1}\right) \sin \left(z_{2}\right) \text { and } w_{2}=\psi\left(z_{1}\right) \sin \left(z_{2}\right),
$$

where

$$
\phi=\frac{k+2}{k} \sin \left(\frac{k z_{1}}{l}\right)-\sin \left(\frac{(k+2)}{l} z_{1}\right)
$$

and

$$
\psi=\cos \left(\frac{k}{l} z_{1}\right)-\cos \left(\frac{(k+2)}{l} z_{1}\right) .
$$

See (4.14). In Sect. 7 we saw that the double cosine series expansion of $\left[w_{i}, w_{j}\right]$ was rather simple, consisting of just a few non-zero terms, so that the computation of $A, B$ and $C$ was easy. For the eigenfunctions in (8.1) this cosine series for $\left[w_{1}, w_{2}\right]$ has an infinite number of non-zero terms, so we use a different technique.

Notice that in (7.5) $A$ has the form

$$
A=A_{1}\left(z_{1}\right)+A_{2}\left(z_{1}\right) \cos \left(2 z_{2}\right),
$$


where $\int_{\Omega} A=0 ; B$ and $C$ have similar structure. It turns out that this is still true for the $A, B$, and $C$ of this section. Observe that if we find a solution in the form (8.3) then we are finished as $\Delta_{N}^{-1}$ restricted to functions with mean zero is injective. Consider further that the image of $\Delta_{N}^{-1}$ satisfies the Neumann boundary conditions so that in (8.3) we have

$$
A_{1}^{\prime}(0)=A_{1}^{\prime}(l \pi)=A_{2}^{\prime}(0)=A_{2}^{\prime}(l \pi)=0 .
$$

Given the form (8.3) one sees from (5.13) that

$$
\begin{aligned}
& 8 a=\frac{1}{\pi l} \int_{0}^{\pi l} A_{1}^{2} d z_{1}+\frac{1}{2 \pi l} \int_{0}^{\pi l} A_{2}^{2} d z_{1} \equiv a_{1}+a_{2} \\
& 4 b=\frac{1}{\pi l} \int_{0}^{\pi l}\left(A_{1} C_{1}+2 B_{1}^{2}\right) d z_{1}+\frac{1}{\pi l} \int_{0}^{\pi l}\left(B_{2}^{2}+\frac{A_{2} C_{2}}{2}\right) d z_{1} \equiv b_{1}+b_{2} \\
& 8 c=\frac{1}{\pi l} \int_{0}^{\pi l} C_{1}^{2} d z_{1}+\frac{1}{2 \pi l} \int_{0}^{\pi l} C_{2}^{2} d z_{1} \equiv c_{1}+c_{2}
\end{aligned}
$$

the last equality being a definition.

Observe that $\left[w_{i}, w_{j}\right]$ has the form (8.3). In fact

$$
\begin{aligned}
& {\left[w_{1}, w_{1}\right]=-\left(\phi \phi^{\prime \prime}+\left(\phi^{\prime}\right)^{2}\right)+\cos \left(2 z_{2}\right)\left(\phi \phi^{\prime \prime}-\left(\phi^{\prime}\right)^{2}\right)} \\
& {\left[w_{1}, w_{2}\right]=-\frac{1}{2}\left(\phi^{\prime \prime} \psi+2 \phi^{\prime} \psi^{\prime}+\phi \psi^{\prime \prime}\right)+\frac{\cos \left(2 z_{2}\right)}{2}\left(\phi^{\prime \prime} \psi-2 \phi^{\prime} \psi^{\prime}+\phi \psi^{\prime \prime}\right)} \\
& {\left[w_{2}, w_{2}\right]=-\left(\psi \psi^{\prime \prime}+\left(\psi^{\prime}\right)^{2}\right)+\cos \left(2 z_{2}\right)\left(\psi \psi^{\prime \prime}-\left(\psi^{\prime}\right)^{2}\right),}
\end{aligned}
$$

whereas applying the Laplacian to (8.3) yields

$$
\Delta A=A_{1}^{\prime \prime}+\left(A_{2}^{\prime \prime}-4 A_{2}\right) \cos \left(2 z_{2}\right) \text {. }
$$

Similarly for $\Delta B$ and $\Delta C$. Clearly (8.6) and (8.7) yield six second order ODE's in the unknown functions $A_{i}, B_{i}$, and $C_{i}$. It is particularly easy to solve the equations for $A_{1}, B_{1}$, and $C_{1}$, the answers being

$$
\begin{aligned}
& A_{1}=-\frac{\phi^{2}}{2}+\alpha_{0} \\
& B_{1}=-\frac{\phi \psi}{2}+\beta_{0} \\
& C_{1}=-\frac{\psi^{2}}{2}+\gamma_{0},
\end{aligned}
$$

where $\alpha_{0}, \beta_{0}$, and $\gamma_{0}$ are constants of integration. The other constants of integration are seen to be zero from the Neumann boundary condition (8.4). From the definition of $\Delta_{N}^{-1}$ we have $\int_{\Omega} A=0$ which implies that $\int_{0}^{\pi} A_{1}\left(z_{1}\right) d z_{1}=0$. So $\alpha_{0}, \beta_{0}$, and $\gamma_{0}$ are determined by

$$
\int_{0}^{\pi l} A_{1} d z_{1}=\int_{0}^{\pi l} B_{1} d z_{1}=\int_{0}^{\pi l} C_{1} d z_{1}=0
$$


Thus

$$
\alpha_{0}=\frac{(k+1)^{2}+1}{2 k^{2}} ; \quad \beta_{0}=0 ; \quad \gamma_{0}=\frac{1}{2}
$$

Using (8.2) and the trigonometric identities (7.4) one can compute (8.8) explicitly we tabulate the results in (8.12) using the notation

$$
\begin{aligned}
z= & z_{1} / l \\
\mathrm{~A}_{1}= & \frac{k+2}{2 k} \cos (2 z)+\frac{(k+2)^{2}}{4 k^{2}} \cos (2 k z)-\frac{(k+2)}{2 k} \cos ((2 k+2) z) \\
& +\frac{1}{4} \cos ((2 k+4) z) \\
B_{1}= & -\frac{1}{2 k} \sin (2 z)-\frac{k+2}{4 k} \sin (2 k z)+\frac{k+1}{2 k} \sin ((2 k+2) z) \\
& -\frac{1}{4} \sin ((2 k+4) z) \\
C_{1}= & \frac{1}{2} \cos (2 z)-\frac{1}{4} \cos (2 k z)+\frac{1}{2} \cos ((2 k+2) z) \\
& -\frac{1}{4} \cos ((2 k+4) z)
\end{aligned}
$$

Now, using (8.5), one computes (noting that $k=1$ is special)

$$
\begin{aligned}
& a_{1}=\frac{5}{16}+\frac{5}{4} \frac{k+1}{k^{2}}+\frac{1}{2} \frac{(k+1)^{2}}{k^{4}}\left(\frac{131}{16} \text { when } k=1\right) \\
& b_{1}=\frac{5}{16}+\frac{5}{8} \frac{k+1}{k^{2}}\left(\frac{63}{16} \text { when } k=1\right) \\
& c_{1}=\frac{5}{16}\left(\frac{3}{16} \text { when } k=1\right) .
\end{aligned}
$$

Next we wish to compute $A_{2}, B_{2}$, and $C_{2}$. The second order ODE's given by (8.6) and (8.7) no longer have such an easy solution as in (8.8). One now has to compute explicitly with (8.2), the RHS of the equations being given by:

$$
\begin{aligned}
\phi \phi^{\prime \prime}-\left(\phi^{\prime}\right)^{2} & =-\frac{2(k+2)}{k}+2 \frac{(k+1)^{2}}{k^{2}} \cos (2 z)-\frac{2}{k^{2}} \cos ((2 k+2) z) \\
\frac{1}{2}\left(\phi^{\prime \prime} \psi-2 \phi^{\prime} \psi^{\prime}+\phi \psi^{\prime \prime}\right)= & \frac{2(k+1)}{k^{2}(k+2)} \sin ((2 k+2) z)-\frac{2(k+1)^{2}}{k^{2}(k+2)} \sin (2 z) \\
\psi \psi^{\prime \prime}-\left(\psi^{\prime}\right)^{2}= & -2 \frac{(k+1)^{2}+1}{k(k+2)}+2 \frac{(k+1)^{2}}{k(k+2)} \cos (2 z) \\
& +\frac{2}{k(k+2)} \cos ((2 k+2) z)
\end{aligned}
$$


The ODE's have the form $A_{2}^{\prime \prime}-4 A_{2}=(8.14)$. The solutions are (where $m=2 k^{2}$ $+4 k+1)$

$$
\begin{aligned}
& A_{2}=\frac{k+2}{2 k}-\frac{k+2}{2 k} \cos (2 z)+\frac{k+2}{2 k m} \cos ((2 k+2) z) \\
& B_{2}=p \sin (2 z)-q \sin ((2 k+2) z)+r f(z)
\end{aligned}
$$

where

$$
\begin{aligned}
& p=\frac{1}{2 k}, q=\frac{k+1}{2 k m}, r=\frac{l}{2 k m}, \text { and } f(z)=\frac{\sinh ((2 z-\pi) l)}{\cosh (\pi l)} \\
& C_{2}=\frac{k^{2}+2 k+2}{2 k(k+2)}-\frac{1}{2} \cos (2 z)-\frac{1}{2 m} \cos ((2 k+2) z) .
\end{aligned}
$$

Note that the constants of integration have been chosen so that the boundary conditions (8.4) are satisfied.

One may now compute using (8.5) and (8.15) that

$$
\begin{aligned}
& a_{2}=\frac{(k+2)^{2}}{16 k^{2}}\left[3+\frac{1}{m^{2}}\right] \\
& c_{2}=\frac{1}{16}\left[1+\frac{2\left(k^{2}+2 k+2\right)^{2}}{k^{2}(k+2)}+\frac{1}{m^{2}}\right] \\
& b_{2}=\frac{3}{16}+\frac{3}{8 k}+\frac{3}{8 k^{2}}+E,
\end{aligned}
$$

where

$$
|E|<\frac{1}{32 k^{2}} .
$$

To obtain this estimate observe that

$$
\begin{aligned}
E= & -\frac{k+2}{16 k m^{2}}+\frac{q^{2}}{2}+\frac{2 r^{2}}{\pi l} \int_{0}^{\pi l} f(z)^{2} d z_{1}+\frac{2 p r}{\pi l} \int_{0}^{\pi l} f(z) \sin (2 z) d z_{1} \\
& -\frac{2 q r}{\pi l} \int_{0}^{\pi l} f(z) \sin ((2 k+2) z) d z_{1} .
\end{aligned}
$$

Next change the variable of integration $z_{1}$ to $z$; note that $f(z), \sin (2 z)$; and $\sin ((2 k+2) z)$ are all odd with respect to the point $z=\frac{\pi}{2}$; observe that $|f(z)|<e^{-2 l z}$ on $\left(0, \frac{\pi}{2}\right)$, and use $|\sin |<1$ to obtain:

$$
|E|<\frac{k+2}{16 \mathrm{~km}^{2}}+\frac{q^{2}}{2}+\frac{r^{2}}{2 \pi l}+\frac{2 r}{\pi l}(p+q) .
$$


It is now routine to obtain the estimate for $E$ in (8.17)

Combining (8.13) and (8.16) yields (for $k \geqq 2$ )

$$
\begin{aligned}
& 8 a=\frac{1}{2}+\frac{2}{k}+\frac{2}{k^{2}}+\frac{(k+2)^{2}}{16 k^{2} m^{2}}+\frac{(k+1)^{2}}{2 k^{4}} \\
& 8 c=\frac{1}{2}+\frac{(k+1)^{2}}{2 k^{2}(k+2)^{2}}+\frac{1}{16 m^{2}} \\
& 4 b=\frac{1}{2}+\frac{1}{k}+\frac{1}{k^{2}}+E .
\end{aligned}
$$

Moreover a simple calculation directly from (5.13) yields

$$
\frac{\alpha}{\beta}=\frac{(k+2)^{2}}{k^{2}+2 k+2} \text {. }
$$

Proposition 8.19. The modal parameters $\mu$ and $v$ fall in region 3 of Fig. 6 . In particular $\mu>1$ and $\frac{1}{\mu}<v<1$.

The result is obtained by using the following estimates when $k \geqq 2$

$$
\begin{aligned}
1+\frac{4}{k}+\frac{5}{k^{2}} & <16 a<1+\frac{4}{k}+\frac{6.3}{k^{2}} \\
1+\frac{2}{k}+\frac{7}{4 k^{2}} & <8 b<1+\frac{2}{k}+\frac{9}{4 k^{2}} \\
0 & <16 c<1+\frac{0.6}{k^{2}} .
\end{aligned}
$$

Note that showing that $v>\frac{1}{\mu}$ is equivalent to showing that $b^{2}-a c>0$. Using the estimates one obtains the result for $k \geqq 2$ and explicitly checks that when $k=1$ the result is also true. In fact for $k=1, \mu=1.0857$ and $v=0.9715$.

\section{Length is the Unfolding Parameter}

In Sect. 6 we described results from singularity theory [4] which enabled us to classify all small, symmetry preserving perturbations of the von Kármán equations near a double eigenvalue by the addition of one new parameter $\sigma$ defined in (6.9). We now show that for clamped boundary conditions the aspect ratio $l$ of the rectangular plate is a physical realization of $\sigma$.

Let

$$
L=\frac{l-l_{0}}{l_{0}},
$$

where $l_{0}$ is the aspect ratio at a double eigenvalue; so $l_{0}=\sqrt{k(k+2)}$ for some positive integer $k$. We will show that, to first order in $L$,

$$
\sigma=\frac{8}{k(k+2)}\left(1-\frac{1}{k^{2}+2 k+2}\right) L
$$


As the coefficient of $L$ in (9.2) is always positive we see that $l$ is the desired realization of $\sigma$. (A similar calculation for simply supported conditions could also be given.)

We now sketch the steps necessary to prove (9.2). The implicit function theorem which gives the existence of the Lyapunov-Schmidt reduction also guarantees that the resulting reduced equation $G$ varies smoothly with $L$. This, along with the fact that $L$ preserves all symmetries in the problem implies, as in (5.11), that

$$
G(x, y, \lambda, L)=\left(\frac{\partial \tilde{V}}{\partial x}, \frac{\partial \tilde{V}}{\partial y}\right)
$$

where

$$
\tilde{V}(x, y, \lambda, L)=a x^{4}+b x^{2} y^{2}+c y^{4}+(d-\alpha \lambda) x^{2}+(e-\beta \lambda) y^{2}+\ldots
$$

and $\ldots$ indicates terms of higher order in $x, y, \lambda$. Here $a, b, c, d, e, \alpha, \beta$ all depend (smoothly) on $L$. Moreover, at $L=0, d=e=0$ while the remaining variables equal their respective counterparts in (5.11). The scaling of (5.20) shows that to first order in $L$ one has

$$
G(x, y, \lambda, L)=\left(x^{3}+\mu x y^{2}-\bar{\lambda} x, v x^{2} y+y^{3}-\left(\bar{\lambda}+\left(\frac{d_{0}}{\alpha}-\frac{e_{0}}{\beta}\right) L\right) \gamma\right)+\ldots,
$$

where $\ldots$ indicates terms of higher order in $x, y, \lambda, L$. Moreover, $\mu$ and $v$ are as in (5.17) and

$$
d_{0}=\frac{\partial d}{\partial L}(0) ; \quad e_{0}=\frac{\partial e}{\partial L}(0) ; \quad \text { and } \quad \bar{\lambda}=\lambda-\frac{d_{0}}{\alpha} L
$$

We claim

$$
\begin{aligned}
& d_{0}=-\left(2 \Delta \omega_{1}+\lambda_{0} \omega_{1}, \frac{\partial^{2}}{\partial z_{1}^{2}} \omega_{1}\right) \\
& e_{0}=-\left(2 \Delta \omega_{2}+\lambda_{0} \omega_{2}, \frac{\partial^{2}}{\partial z_{1}^{2}} \omega_{2}\right),
\end{aligned}
$$

where $\omega_{1}, \omega_{2}$ are the eigenfunctions (8.1) and $\lambda_{0}$ is the applied force at the double eigenvalue. Thus by (4.11), we have

$$
\lambda_{0}=2+\frac{k}{k+2}+\frac{k+2}{k} .
$$

Assuming this claim one makes a calculation involving integrals of the type considered in Sect. 8 to obtain

$$
d_{0}=0 \text { and } e_{0}=-4\left(\frac{k+1}{k(k+2)}\right)^{2}
$$

Finally (9.2) follows from the observation that

$$
\beta=\left\|\frac{\partial \omega_{2}}{\partial z_{1}}\right\|^{2}=\frac{k^{2}+2 k+2}{2 k(k+2)} .
$$


We now prove the claim. The basic idea is to first change variables so that the von Kármán equations are computed on a common domain for all $L$. Let

$$
\Omega_{l}=\left\{0 \leqq z_{1} \leqq l \pi, 0 \leqq z_{2} \leqq \pi\right\} .
$$

Consider the change of variables

$$
\left(z_{1}, z_{2}\right) \rightarrow\left(m z_{1}, z_{2}\right) \text { of } \Omega_{l_{0}} \rightarrow \Omega_{l},
$$

where $m=l / l_{0}$, the potential function (3.1) has the form

$$
V(\omega, \lambda, m)=\frac{1}{2}\left\|\frac{1}{m^{2}} \frac{\partial^{2} \omega}{\partial z_{1}^{2}}+\frac{\partial^{2} \omega}{\partial z_{2}^{2}}\right\|^{2}-\frac{\lambda}{2}\left\|\frac{1}{m} \frac{\partial \omega}{\partial z_{1}}\right\|^{2}+Q(\omega),
$$

where $Q$ is a quartic expression in $\omega$. As $Q$ only effects the quartic terms in (9.4) it does not enter the calculation of $d_{0}$ and $e_{0}$.

Observe that $m-1=L$. Thus to compute $\frac{\partial V}{\partial L}$ at $L=0$, we need to compute

$$
\frac{\partial V}{\partial m}(\omega, \lambda, l)=-2\left(\frac{\partial^{2} \omega}{\partial z_{1}^{2}}, \Delta \omega\right)+\lambda\left\|\frac{\partial \omega}{\partial z_{1}}\right\|^{2} .
$$

Of course, the evaluation of $d_{0}$ and $e_{0}$ is at $\lambda=\lambda_{0}$. As in Sect. 5 the calculation of $G$ to lowest order does not involve the implicitly defined function $W$ [of (5.7)]. So the Taylor expansion of $\tilde{V}$ to lowest order is the same as the Taylor expansion of

$$
\tilde{\tilde{V}}(x, y, \lambda, m)=V\left(x \omega_{1}+y \omega_{2}, \lambda, m\right) \text {. }
$$

Using (9.3) and extracting the coefficient of $x^{2}$ and $y^{2}$ from (9.15) and (9.14) yields (9.7) as desired.

Acknowledgement. We are grateful to Ed Reiss for calling our attention to Stein's paper [9]. Our efforts to understand that paper were greatly aided by a number of stimulating conversations with Wayne Dickey. Jeff Holder most kindly corrected a number of mistakes in the original version of Sect. 8.

\section{References}

1. Bauer, L., Keller, H.B., Reiss, E.L.: Multiple eigenvalues lead to secondary bifurcation. SIAM J. Appl. Math. 17, 101-122 (1975)

2. Chow, S.N., Hale, J.K., Mallet-Paret, J. : Application of generic bifurcations. I, II. Arch. Rat. Mech. Anal. 59, 159-188 (1975); 62, 209-235 (1976)

3. Golubitsky, M., Schaeffer, D. : A theory for imperfect bifurcation via singularity theory. Commun. Pure Appl. Math. 32, 21-98 (1979)

4. Golubitsky, M., Schaeffer, D. : Imperfect bifurcation in the presence of symmetry. Commun. Math. Phys. 67, 205-232 (1979)

5. Magnus, R., Poston, T.: On the full unfolding of the von Kármán equations at a double eigenvalue. Battelle Math. Report No. 109. Geneva 1977

6. Matkowsky, B.J., Putnick, L.: Multiple buckled states of rectangular plates. International J. Nonlin. Mech. 9, 89-103 (1973)

7. Sattinger, D.H.: Group representation theory, bifurcation theory, and pattern formation. SIAM J. Math. Anal. 8, 179-201 (1977)

8. Stein, M.: The phenomenon of change in buckle patterns in elastic structures. NASA technical report R-39 (1959)

9. Stein, M.: Loads and deformations of buckled rectangular plates. NASA technical report R-40 (1959)

10. Stoker, J.J.: Nonlinear elasticity. New York: Gordon and Breach 1968

Communicated by J. Moser

Received January 16, 1979 